\title{
LOS GUARANÍ Y SU ORIENTACIÓN EN EL ESPACIO
}

\author{
The guarani and spatial orientation
}

\begin{abstract}
Angélica Otazú*
\section{Resumen}

El presente trabajo pretende abordar la experiencia de los guaraní-kaiowá referente a su orientación en la selva y señalar, brevemente, algunas similitudes con los datos recopilados por los misioneros que entraron en contacto con los guaraní durante la colonia, especialmente, los de la Compañía de Jesús, quienes registraron importantes informaciones sobre la cultura guaraní, incluidas la fauna y la flora del Río de la Plata, entre otras regiones. La convivencia con la naturaleza les ha facilitado a los nativos orientarse, convenientemente, en el medio que le rodea, el bosque. Según los relatos de los kaiowá, durante siglos han logrado desenvolverse en perfecta armonía con la naturaleza y podrían desplazarse libremente, sea para cazar animales o recolectar frutas silvestres, de esta manera, atravesaban vastos territorios sin barrera alguna, muy diferente a la situación actual de muchos nativos tanto del Mato Grosso do Sul como los de Paraguay. La dinámica del viento, yvytu les permitía orientarse en medio de la selva; yvytu oipejuveha koty, el viento dominante, el otro medio infalible de orientación es el sol, kuarahy pyti'a, kuarahy sẽmba, oriente; kuarahy reike, poniente. Para circular por el monte dejaban trazos, cortando ramas de las plantas de modo que sirvan de señalización para volver a la casa. Habilidades que la sociedad, considerada moderna, no lo comprende o simplemente, desconoce. La orientación y habilidad, incomparables, que poseen los nativos nos invita a considerar la riqueza natural que ellos custodian y que están en permanente lucha para frenar la deforestación. Los habitantes más antiguos de estas zonas priorizaban el uso equilibrado de los recursos naturales. Por ejemplo, para sembrar y cosechar poseían un calendario lunar. Lógicamente, la división del tiempo, ára se basa en las fases de la luna, jasy ra'y, luna nueva; jasy renyhẽ, luna llena.
\end{abstract}

$$
<\text { Guaraní }><\text { Kaiowá }><\text { Historia }><\text { Cosmovisión }>
$$

\begin{abstract}
This paper aims to address the experience of the Guarani-Kaiowá concerning their means of orientation in the jungle, and to briefly highlight some points of comparison with information collected by the missionaries, who came into contact with the Guarani during colonial times. Coexistence with nature has allowed the natives to orient themselves within their surrounding environment, the rain forest. In the past, they crossed vast territories, moving freely without being impeded by any man-made barrier; quite differently from now, for the numerous natives of both Mato Grosso do Sul and Paraguay. They could find their way perfectly while always respecting nature. The dynamics of the wind Yvytu enabled them to orientate themselves in the jungle; Yvytu oipejuveha koty, is the prevailing wind. The other reliable means of guidance is the sun, Kuarahy pyti'a or Kuarahy Semba, for finding the east; Kuarahy reike, for finding the west. To move through the bush, they left marks by cutting plant branches, to serve as signs which showed the way to return home. Skills that our society, considered modern, do not understand or simply do not recall. Such incomparable orientation skills, that the natives still possess today, compel us to consider
\end{abstract}

* Universidad Católica “Nuestra Señora de la Asunción”-CONACYT / Paraguay. potykuru@hotmail.com 
the importance of preserving natural reserves and to bear in mind that they are presently immersed in a constant struggle as they try to curb deforestation. They are the people who have inhabited these areas the longest and that have always prioritized the balanced use of natural resources. For example, to plant and harvest they use a lunar calendar. Consequently, the division of time is based on the phases of the moon, jasy ra'y, new moon; jasy renyhẽ, full moon.

$<$ Guarani $><$ Kaiowa $><$ History $><$ Worldview $>$

Recibido: 25/09/2016 //Aceptado: 10/12/2016

\section{A modo de introducción}

El contacto con la naturaleza, durante miles de años, les había proporcionado a los nativos notables conocimientos y experiencias que despertaron en ellos el respeto hacia los seres vivos y el interés por conocer las propiedades de las plantas. En la selva aprendieron todo tipo de mecanismo de sobrevivencia, en permanente búsqueda de la felicidad, vy'a, que implica, sobre todo, vivir libremente y en sintonía con la tierra, el universo. Los más ancianos señalaron que la vida en los grandes bosques tiene sus ventajas, por el hecho de servir como vivienda y asiento de las personas, animales y plantas. Aseveran que la selva daba vida y proporcionaba el sustento de cada día, y evocan sin cesar el bienestar que producía la frescura del bosque (ka'aguy ro'ysã). Además, están convencidos de que la selva proveía la lluvia, mejor dicho, que la lluvia depende de ka'aguy, por lo tanto, si no hay bosque no habrá lluvia. En cierta manera, la selva se constituía en una especie de base de resistencia y supervivencia, a la vez, pero ese espacio fue transformándose desde hace décadas, dado que los pocos montes que quedan en las fronteras entre Paraguay y Brasil (Sur de Mato Grosso) fueron destruidos casi por completo. El presente trabajo aborda el tema de la orientación desde dos perspectivas diferentes, a saber, la información obtenida entre los kaiowá del Mato Grosso del Sur, durante la elaboración de un diccionario temático y etnológico (20132015) y el estudio bibliográfico sobre otros grupos de la familia tupí-guaraní. Con el fin de rescatar un aspecto de la historia y la experiencia de varios siglos de un determinado grupo de personas, se presenta algunos datos que hacen referencia a la señalización vial en la selva, bajo diversos elementos, como las plantas, los árboles; el curso de agua, los ríos; el viento; los astros y las constelaciones. Por último, se señala algunos datos relacionados a la orientación que figuran en el Tesoro de la Lengua guaraní (1639) y se expone brevemente la similitud existente entre las nociones de orientación en el espacio y las prácticas tradicionales de algunos grupos de la familia tupi-guaraní, que perduran hasta la actualidad. Se aclara que la traducción de las frases en kaiowá es de la autora. 


\section{Señalización vial en las selvas guarani}

\section{a) Referente a las plantas}

Los nativos mencionaron numerosos mecanismos muy antiguos referentes a la señalización vial en la selva y muchos de ellos tienen relación con las plantas: Yvyra ho'a va'ekue rupi oĩ tape, a través de los árboles caídos se abren los senderos. Amopẽ yvyra rakangue aikuaa haguã che rape, cortar ramas de los árboles como señalización; Ka'a iñana kuéry aipe 'ávy akua, ir abriendo camino entre malezas. Opa opa tape, significa, las dificultades del camino. Jaiguy rupi aiko, paso por debajo de los matorrales. Iñana e' $\tilde{y}$ rupi ahasa, fui por donde hay trazos o bien, por donde los árboles fueron derribados. Che ropa ka 'a roguety rupi, perdí la orientación en el bosque. Tape ho 'ysã porãha rupi aju, escogí el camino más fresco.

Asimismo, los nativos remarcaron que en la selva existía una especie de horario para desplazarse: Nde reguatái arã pyhare ka'aguýre, no es recomendable andar de noche por la selva. Ka 'a rupi rehasapávy, atravesar la selva. Che ka 'aru reíma, me tomó la noche por el camino. Sin embargo, si hay urgencia de llegar a algún lugar, se dice, ekua ta nde ka'aru jepe rehóvy, aunque caiga la noche es menester que continúe tu camino. $\mathrm{El}$ arte de desenvolverse por el bosque se nota en la capacidad de caminar una distancia considerable y encontrar el camino de vuelta a casa y este camino es denominado Tape jevyha. Ejemplo: Tape jevyha rupi aju, he tomado el camino de vuelta. Vale mencionar también, que en el Tesoro de la Lengua Guaraní se ha recopilado algunas frases relativo al camino en la selva: $k a$ 'a juru, entrada, o callejón de monte (Montoya, 2011; 230).

\section{b) Referente al curso de agua}

Otra referencia importante para transitar por los senderos constituye el curso de agua, como los arroyos y los ríos: y guejypaha rupi ahasa, fui por el camino que lleva al agua, cuesta abajo. Para indicar una dirección se puede decir, Y piaha koty oĩ tape, camino que conduce al río o camino al arroyo. Rehasa arã peteĩ y syry reguahẽ haguã og gusúpe, debes atravesar un arroyo para llegar a la casa de rezo, literalmente, casa grande, lugar donde se realizan las danza rituales, referencia interesante en un Tekoha. Y mboypýri oĩ che taita róga, allende del arroyo está ubicada la casa de mi padre.

Se pudo recopilar también otras informaciones referentes al camino, expresadas de las siguientes maneras: Che rape rupi mante aha, frecuento el mismo camino, mi camino habitual. Upe tape jehe 'a rupi aháta, fui por dos caminos que tienen un punto de conjunción. Dese luego, abundan frases que dejan entrever los peligros frecuentes que implicaban desplazarse por los senderos de la selva: Pe tape jeporu e'ỹ rupi oho mbó $i$, por los caminos no transitados, normalmente, frecuentan las víboras.

Además, se constata que la experiencia de varios siglos les ha otorgado a los nativos la habilidad de encontrar siempre la salida o el retorno a casa, en la otrora frondosa selva latinoamericana; a continuación, se expone una frase que explica esta afirmación: Upe tape rejevyha nde rekohápe heta õ̃, hay muchos caminos de retorno. Siempre se consigue encontrar el camino de retorno a casa. Se expresa, asimismo, los 
caminos desconocidos, Tape ndajaikuaa kuaáiva, los caminos que no conocemos, o los caminos dificultosos, difícil de transitar. Es menester resaltar que hay un camino que se transita gracias a la práctica concerniente a la espiritualidad, específicamente, para alcanzar el yvy marãe '́y, la tierra sin mal.

\section{c) Referente a la dinámica del viento}

Gracias a la dinámica del viento los nativos se conducían por en medio de la selva; curiosamente, quedó grabada en la memoria de los kaiowá hablantes uno de los elementos más importante del ser humano, poder orientarse naturalmente en cualquier ambiente, y esto sucedió por medio de la conservación de la propia lengua, que transmite toda una cultura, las costumbres, las formas de pensar y los medios de sobrevivencia. En ese sentido tenemos una frase que define su tradicional forma de orientación, yvytu oipeju hatãveha rupi reguata arã, dejarse guiar por el viento dominante. Yvytu ñande reraha, el viento es nuestra guía. Yvytu atã jave neresẽ arãi, cuando el viento sopla fuerte mejor no salir. En el Tesoro de la Lengua guaraní se ha registrado también algunas frases referentes al viento: yvytu ombokatu ova ka'a, el viento ha derribado mucho monte (Montoya, 2011; 246). Yvytu katu ete, gran viento; viento favorable; viento fresco (Montoya, 2011; 655). Yvytu ai, tempestad (Montoya, 2011; 655). Los kaiowá, por su parte, trataron de resumir la descripción de la selva en una frase: Yvyra heta hápy oĩ ka'aguy, selva significa donde hay muchos árboles. Con esta definición, probablemente, querían indicarnos que la generación actual ya difícilmente conocería el sentido y la importancia de la selva, a causa de la masiva deforestación.

\section{d) Referente a los astros y constelaciones}

La posición solar es una referencia determinante en la orientación en las grandes selvas y para calcular la hora. Kuarahy sẽmba, kuarahy pyti'a, oriente. Estas dos frases son empleadas indistintamente por los nativos para indicar una dirección o localizar un lugar específico; kuarahy reike, poniente. Ka'aru he'ise ohóma kuarahy, es tarde, ya el sol se asoma en el poniente. Asimismo, sirve de indicador de tiempo para concertar una cita con alguien, kuarahy reike rupi ajúne ne rendápe, cuando el sol se asome en el poniente iré junto a ti. Áry resakã porã kuarahy ohesape jave, cuando el sol alumbra el día es más claro. Che ru pa ’i kuara, mi padre pa'i kuara (kuarahy). Según la mitología kaiowá pa'i kuara es kuarahy, sol. Oike jave kuarahy ereikepáma kotýpe, a la puesta del sol ya debes encerrarte en la habitación. El movimiento solar diario es empleado como referencia o indicador de dirección: Kuarahy resẽ kotyo oho, se marchó en dirección al oriente. Kuarahy osẽhápy, inicio de la división del día.

Las fases lunares constituyen, pues, los meses, jasy, así tenemos jasyho, un mes. En cambio, el año es determinado por el invierno, peteĩ ro 'yho: un año. En uno de los documentos más importantes para la etnografía guaraní, como el Tesoro de la Lengua Guaraní de Ruiz de Montoya (1639) aparecen informaciones sistemáticas sobre la división del tiempo basada en las fases lunares. De acuerdo a los registros de Montoya 
jasy, luna, equivale a un mes; jasy ypýramo, al principio del mes; jasy ojeova ynyhe mbotáramo mediado del mes; jasy kañỹ kakáramo, al fin del mes (Montoya, 2011; 203). Jasyy asajéramo, está la luna en medio cielo (Montoya, 2011; 319).

Para indicar la duración de una determinada acción en un periodo concreto decían: Peteĩ jasy añemboty, un mes me detuve (Montoya, 2011; 602). Algunas de las formas de explicar cuanto tiempo se ausentarán de sus casas eran: Peteĩ jasy aiko evapóne, un mes estaré allá; mokõi jasy kua vire ajevýne volveré de aquí a dos meses (Montoya, 2011; 203). Siguiendo con lo recopilado en el Tesoro encontramos que para confirmar la fecha preguntaban: Mbovy ára piko jareko? mbovy jasy árareve piko ñañ? ¿Cuántos son del mes? (Montoya, 2011; 6).

Las fases de la luna se describen en guaraní de la siguiente manera, Jasy pyahu, luna nueva; jasy kakuaa, creciente; jasy pytũ angaivo, menguante (Montoya, 2011; 203); jasy ypytũmba vai menguante de luna (Montoya, 2011; 203); jasy ei, hace luna; jasy oikie, ponerse la luna; jasy ova ynyhẽ; jasy ova guasu, luna llena; jasy rova pembai; hovápe jasy, muy menguada luna, o cuando nace (Montoya, 2011; 203). Hovapysẽ jasy asoma la luna (Montoya, 2011; 173).

\section{Similitud entre los conocimientos kaiowá, tupinambá y los datos registrados por Montoya}

En la cultura kaiowá, las épocas del año para sembrar y cosechar se basaban, principalmente, en las fases lunares, jejaty, sembrar, cultivar y cosechar, ñemono'õ. Asimismo, es interesante poner de relieve los estudios realizados por los investigadores en cuanto a la utilización de la astronomía por parte de los nativos del Brasil, especialmente los Tupinambá "Os indígenas utilizavam a astronomia principalmente para a agricultura. Dessa maneira associavam as estações do ano e as fases da Lua com a biodiversidade local, para determinarem a época de plantio e da colheita, bem como para a melhoria da produção e o controle natural das pragas" (Guerreiro, 2016; [s. p.]).

Es importante señalar, a propósito de la siembra, en el Tesoro se menciona que el tiempo propicio depende de la dirección en la que sopla el viento: Yvytu pu'ã, viento leste, cuando corre dicen que es tiempo de sembrar (Montoya, 1639; 170:2; 2011; 655) ${ }^{1}$. Puesto que, los vientos son designados en relación con las direcciones en las que soplan, en guaraní el viento este se denomina yvytu pu'ã. Por otro lado, los kaiowá aseguran también que las fases lunares tienen una influencia en la realización de la cura, en ese sentido, enumeraron algunas enfermedades que se deben tratar en la luna nueva para que el remedio sea más eficaz, jasy ra’y jave ereipohano va'erã. Además, explican que el comportamiento de algunos animales depende de las fases lunares, por ejemplo, Ajaku, peteĩ ynambu guasu, okéva yvýpy jasy ra'y ramo, hay una especie de perdiz que en la luna nueva duerme en el suelo; ha okéva yvate jasy tuicha vévo, en la creciente duerme en las ramas de los árboles.

1 Ver Caballos. A. 2012; 119. 
Yvag jasy tata rendypu cielo estrellado (Montoya, 2011:164) jasy tata, estrellas. Los kaiowá prefieren Jasy tataendy para denominar la estrella. En cambio, en el Tesoro de Montoya encontramos diferentes informaciones sobre las denominaciones de las estrellas. jasy tata guasu lucero. Jasy tata kurusu crucero del cielo; jasy tata veve; jasy tata ho'a cometa; exhalación; jasy tata mimbi, reverberar las estrellas; jasy tata piriri, centellear

Eichu las siete cabrillas [Pléyades, cúmulo estelar en la constelación del Toro]; abejas negras (Montoya.1639, 124v:1; 2011; 118). Según un misionero capuchino del siglo XVII, los Tupinambá identificaban también a las pléyades con las abejas negras, eichu,

"los Tupinambá conheciam muito bem o aglomerado estelar das Plêiades e o denominavam Eixu (Vespeiro). Quando elas apareciam afirmavam que as chuvas iam chegar, como chegavam, efetivamente, poucos dias depois. Como a constelação Eixu aparecia alguns dias antes das chuvas e desaparecia no fim para tornar a reaparecer em igual época, eles reconheciam perfeitamente o intervalo de tempo decorrido de um ano a outro"2.

Otras descripciones de la luna registradas por Montoya que revelan los conocimientos nativos son los siguientes: jasy amandáu mancha de agua en la luna; jasy iñamandáu; jasy havẽ denota agua la luna; jasy havẽramo oky en estando pálida la luna llueve; jasy pyrãramo yvytu peju; jasy pytãngamo yvytu peju, estando encendida la luna corre viento; jasy tĩ ngatúramo irui katu ára, estando blanca la luna está asentado el tiempo; yvag mongyháva jasy tata, el ornato del cielo son las estrellas (Montoya, 2011; 319).

\section{A modo de conclusión}

Los relatos de los guaraní kaiowá actuales demuestran que conservan gran parte de los legados de sus ancestros, tanto en el manejo de los vocablos como en la práctica. Motivo suficiente para que los kaiowá sigan luchando por sus territorios y resistan a diversos tipos de atropellos cometidos contra su población. Es precisamente en la sabiduría de sus antepasados que encuentran el sentido de su lucha. Es evidente, que la cultura no desaparece mientras haya un pueblo que la promueve y busca nuevos caminos para innovar las prácticas tradicionales. Ellos educan a sus hijos acorde a sus creencias, de esta manera, transmiten a sus hijos y nietos la importancia del uso equilibrado de los recursos naturales, les enseña cómo emplear el tiempo en las dos estaciones, aparentemente, las más importantes del año áry haku, verano y áry ro'y, invierno. Aunque, el calendario kaiowá contempla más parcelación en las estaciones del año que en el nuestro, pues se basa en los acontecimientos que marcan su vida. Incluyen en su calendario hasta el proceso del crecimiento de las plantas, desde el cultivo hasta la celebración y fiesta de la cosecha. Asimismo, consideran las diversas estaciones de las frutas maduras, yva aju.

2 D’ Abbeville. En: Alfonso, G. 2016. As constelações indígenas brasileiras. Observatórios virtuais - VITAE. 
En resumen, los kaiowá pertenecen a una gran familia llena de historia y rica en sabiduría, de la cual tenemos mucho para aprender, fundamentalmente, en lo referente al cuidado de la naturaleza y el uso equilibrado de los recursos naturales. Con su estilo de vida y filosofía nos proporcionan una propuesta del desarrollo sostenible basada en el vivir en armonía con la tierra, yvy y el cosmos, yvága. Para lograr la felicidad, vy'a insisten en que la persona debe estar bien consigo misma y con la comunidad, tekoha, siempre en conexión con la naturaleza, sintonizar con la tierra, pues, yvy oñe 'ée, la tierra tiene un mensaje para nosotros.

\section{Referencias bibliográficas}

D’Abbeville, C. 1612. «Histoire de la mission de pères capucins en l'Isle de Maragnan et terres circonvoisines». En: Alfonso, G. As Constelações Indígenas Brasileiras. Observatórios virtuais - VITAE. Disponible en: http://www.telescopiosnaescola. pro.br/indigenas.pdf

Caballos, A. 2012. Etnografía Guarani en Ruiz de Montoya. Granada: Universidad de Granada.

Guerreiro, I. Astronomia Indígena na Amazônia antecede Galileu, diz pesquisador. Disponible en: http://portalamazonia.com/noticias-detalhe/ciencia-etecnologia/indigenas-da-amazonia-estudavam-astronomia-antes-de-galileu-dizpesquisador/. Consultado 30.04.16 17:15

Montoya, A. 1639. 2011. Tesoro de la Lengua Guaraní. Asunción: CEPAG. 